\title{
Mediterranean Diet and Naltrexone/Bupropion
} Treatment for Weight Loss in Overweight and Obese Breast Cancer Survivors and Non-Cancer Participants: A Pilot Randomized Controlled Trial

This article was published in the following Dove Press journal:

Diabetes, Metabolic Syndrome and Obesity: Targets and Therapy

\section{A-Ra Cho' \\ Won-Jun Choi $\mathbb{1 D}^{2}$ \\ Yu-Jin Kwon (D) \\ Hye Sun Lee $\mathbb{D i D}^{3}$ \\ Sung Gwe Ahn (iD) ${ }^{4}$ \\ Ji-Won Lee $\mathbb{D}^{5}$}

'Department of Family Medicine, Yong-in Severance Hospital, Yonsei University College of Medicine, Yong-in 16995, Republic of Korea; ${ }^{2}$ Department of Medicine, Graduate School, Yonsei University, Seoul 03722, Republic of Korea; ${ }^{3}$ Biostatistics Collaboration Unit, Department of Research Affairs, Yonsei University College of Medicine, Seoul 06273, Republic of Korea; ${ }^{4}$ Department of Surgery, Gangnam Severance Hospital, Yonsei University College of Medicine, Seoul 06273, Republic of Korea; ${ }^{5}$ Department of Family Medicine, Gangnam Severance Hospital, Yonsei University College of Medicine, Seoul 06273, Republic of Korea
Introduction: The objective of this pilot randomized controlled trial was to investigate the combined effect of a Mediterranean diet and naltrexone/bupropion treatment on body weight, metabolic parameters, and quality of life in overweight or obese breast cancer survivors.

Methods: Forty-four breast cancer survivors were randomly assigned to receive the Mediterranean diet plus naltrexone/bupropion medication (breast cancer survivor MeDiet $+\mathrm{NB}$ group) or the Mediterranean diet alone (breast cancer survivor MeDiet-only group) Twenty-eight age-matched non-cancer patients were instructed to consume the Mediterranean diet plus naltrexone/bupropion medication (non-cancer MeDiet+NB group). After the 8-week intervention, changes in body weight, metabolic parameters, nutrient intake, and quality of life of the three groups were assessed.

Results: Significant weight loss of $2.8 \mathrm{~kg}$ was noted for the breast cancer survivor MeDiet +NB group, $1.8 \mathrm{~kg}$ for the breast cancer survivor MeDiet-only group, and $2.5 \mathrm{~kg}$ for the noncancer MeDiet+NB group after 8 weeks $(P<0.05$ versus baseline by Wilcoxon's signed-rank test). All three groups also exhibited significantly lower fasting glucose, insulin, and homeostasis model assessment of insulin resistance levels $(P<0.05)$. Quality of life as assessed by self-reported questionnaires showed improvement in all participants $(P<0.05)$. However, there were no significant differences of changes in body weights, metabolic parameters, and quality of life among the three groups or between the MeDiet+NB and MeDiet-only groups. Conclusion: We found that the Mediterranean diet, with or without naltrexone/bupropion treatment, facilitates weight loss, improves metabolic parameters, and increases quality of life. The combination of the Mediterranean diet with naltrexone/bupropion treatment did not produce superior changes when compared to the Mediterranean diet alone.

Trial Registration: This trial was retrospectively registered on 10 July 2018 as NCT03581630 at ClinicalTrials.gov (https://clinicaltrials.gov/ct2/show/NCT03581630).

Keywords: Mediterranean diet, naltrexone/bupropion, obesity, breast cancer survivors

\section{Introduction}

Breast cancer is a leading cause of cancer among women worldwide and the second most common cancer among women in Korea. From 1993 to 2015, the 5-year survival rate for breast cancer patients increased from $78.0 \%$ to $92.3 \%$ in Korea, ${ }^{1}$ indicating the increased importance of health care for survivors. Overweight or obesity is associated with a higher risk for cancer recurrence and mortality among breast cancer survivors. ${ }^{2,3}$ Furthermore,
Correspondence: Ji-Won Lee Department of Family Medicine, Gangnam Severance Hospital, Yonsei University College of Medicine, 21I

Eonju-ro, Gangnam-gu, Seoul 06273,

Republic of Korea

Tel +82220193482

Email indi5645@yuhs.ac
Diabetes, Metabolic Syndrome and Obesity: Targets and Therapy 2020:13 3325-3335

3325

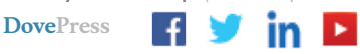


obesity is associated with psychosocial problems, a low quality of life, and increased metabolic risk factors. ${ }^{4,5}$ Thus, the American Cancer Society and the American Society of Clinical Oncology recommend that cancer survivors achieve and maintain a healthy weight. ${ }^{6}$ Recent studies have begun examining the effect of weight loss intervention in overweight or obese breast cancer survivors. ${ }^{7,8}$ However, the bulk of these studies were conducted only in Western countries, and few have been conducted in Asian countries.

Weight loss intervention to address overweight or obesity should include comprehensive lifestyle modifications, and drug treatment for obesity is recommended as an adjunctive therapy. ${ }^{9}$ The Mediterranean diet, recently known as an effective alternative to low-fat or low-carbohydrate diets in terms of weight loss, is associated with a significant reduction in cardiovascular disease and total mortality, as well as body weight. ${ }^{10,11}$ Moreover, an updated meta-analysis pooling seven cohort studies reported an inverse association between the incidence of breast cancer and adherence to a Mediterranean diet. ${ }^{12}$ In a secondary analysis of the PREDIMED (Prevención con Dieta Mediterránea) clinical trial, individuals consuming a Mediterranean diet supplemented with extra-virgin olive oil had a reduced incidence of breast cancer. ${ }^{13}$ These findings suggest that the Mediterranean diet would be an effective dietary intervention for overweight or obese breast cancer survivors.

With regard to pharmacological therapies to improve weight loss, the combination of the opioid receptor antagonist naltrexone and the norepinephrine-dopamine reuptake inhibitor bupropion (Contrave; Orexigen, La Jolla, CA, USA) was recently approved and shows long-term safety and efficacy. Bupropion's anorectic effect is the result of the stimulation of hypothalamic pro-opiomelanocortin neurons, whereas naltrexone blocks opioid-mediated autoinhibition of these cells. ${ }^{14}$ In addition, low-dose naltrexone inhibits tumor progression by upregulating the expression of opioid growth factor and its receptor. $^{15,16}$ Furthermore, bupropion has been shown to improve sexual function in breast cancer patients. ${ }^{17,18}$ Therefore, the combination of naltrexone and bupropion may be beneficial for not only non-cancer obese people but also breast cancer survivors.

The aim of our study was to investigate the effect of the Mediterranean diet and naltrexone/bupropion treatment on body weight, metabolic parameters, and quality of life in breast cancer survivors who are overweight or obese. We also evaluated whether obesity drugs have an additional effect compared to the Mediterranean diet alone and whether there are any differences in breast cancer survivors, compared to non-cancer obese people.

\section{Methods}

\section{Study Design}

This 8-week, three-arm parallel-group, randomized controlled trial was conducted at Gangnam Severance Hospital (Seoul, South Korea) from July 2017 to July 2018. The study design and experimental protocol were approved by the Institutional Review Board of Gangnam Severance Hospital (IRB number 3-2017-0097) in accordance with the Declaration of Helsinki. Written informed consent was obtained from each participant before screening and data collection. This trial was retrospectively registered at ClinicalTrials.gov (number NCT03581630).

\section{Study Participants}

Breast cancer survivors and age-matched non-cancer patients were recruited via poster and online advertisements at Gangnam Severance Hospital. All volunteers were initially screened by telephone. Women aged 20-65 years were eligible for inclusion if they had a body mass index $(\mathrm{BMI})>25.0 \mathrm{~kg} / \mathrm{m}^{2}$ (obese for Asian populations, according to the World Health Organization ${ }^{19}$ ) or a BMI $>23.0 \mathrm{~kg} / \mathrm{m}^{2}$ (overweight for Asian populations) in the presence of at least one metabolic risk factor (waist circumference $\geq 80 \mathrm{~cm}$, triglyceride $\geq 150 \mathrm{mg} / \mathrm{dL}$, highdensity lipoprotein [HDL] cholesterol $<50 \mathrm{mg} / \mathrm{dL}$, fasting glucose $\geq 100 \mathrm{mg} / \mathrm{dL}$, blood pressure $\geq 130 / 85 \mathrm{mmHg}$, or a diagnosis of hypertension, type 2 diabetes mellitus, or dyslipidemia currently controlled with medications). Participants who were diagnosed with breast cancer stage I-III and completed cancer treatment, including surgery, adjuvant chemotherapy, radiotherapy, and/or hormonal therapy, were included in the breast cancer survivor group.

Participants who met any of the following criteria were excluded: uncontrolled hypertension, hepatic or renal disease, significant cardiovascular disease or stroke, history of seizures, serious psychiatric illness (such as bipolar disorder, schizophrenia, bulimia, anorexia nervosa, or suicidal ideation), taking medications such as monoamine oxidase inhibitors, opioid-containing medications, other naltrexone- or bupropion-containing medications, or tamoxifen, history of alcohol abuse or dependence, current smokers or use of nicotine replacement products in the previous 6 months, or women who were pregnant or breastfeeding. Participants with a history of breast cancer were excluded if they had 
experienced cancer recurrence or metastasis. Women of childbearing age were required to use effective contraception throughout the study period and up to 30 days after discontinuation of the study drug.

\section{Randomization}

Breast cancer survivors were randomly assigned to receive the Mediterranean diet plus naltrexone/bupropion medication (MeDiet+NB group) or the Mediterranean diet alone (MeDiet-only group). Participants were randomized using a computer-generated random number sequence with a block size of four. An independent statistician generated the allocation sequence, and the study coordinator assigned the participants to interventions in chronological order as the participants enrolled. Only outcome assessors were blinded to group allocation.

\section{Intervention}

\section{Mediterranean Diet}

All participants participated in the Mediterranean diet intervention and received dietary training from professional nutritionists at the baseline visit and samples of a Mediterranean diet for three times during the trial. To assess adherence to the Mediterranean diet, a 13-item questionnaire developed by the PREDIMED study group that modified the validated Mediterranean Diet Adherence Screener was used. ${ }^{20}$ However, given the study population and evidence linking alcohol consumption with cancer, ${ }^{21}$ the item for wine consumption was excluded. The resulting modified scores ranged from 0 to 13, with scores higher than 9 defined as high adherence to the Mediterranean diet. Participants were educated to increase the scores by the following diet modifications: (i) abundant use of olive oil, sesame oil, or perilla oil for cooking and dressing; (ii) increased consumption of vegetables, fresh fruits, legumes, fish, nuts, white meat, and sauce made with tomato, onion, garlic, or olive oil; and (iii) avoidance of red or processed meat, butter or cream, sugarsweetened beverages, and commercial sweets or pastries. Details of the modified Mediterranean diet score questionnaire are provided in the online supporting material (Table

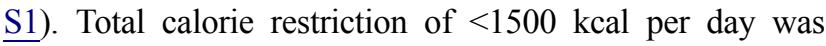
advised, which is a $500 \mathrm{kcal}$ deficit from the daily recommended calories calculated according to the method presented by the Korean Diabetes Association. ${ }^{22}$ All participants were encouraged to communicate with the study researchers via mobile messenger by submitting their daily diet photographs and Mediterranean diet scores and receiving feedback (Figure S1).

\section{Naltrexone/Bupropion Medication}

Breast cancer survivors assigned to the MeDiet $+\mathrm{NB}$ group and all non-cancer patients were instructed to take naltrexone/ bupropion. Participants took one extended-release tablet containing $8 \mathrm{mg}$ naltrexone and $90 \mathrm{mg}$ bupropion each morning for the first week. Then if tolerable, they increased the daily dose to two tablets, one in the morning and one in the evening for the next 7 weeks. Drug compliance was measured by the remaining pill count at week 1,4 , and 8 . Noncompliant participants (compliance rate of less than $80 \%$ ) were discontinued from the study.

\section{Outcome Measurements}

Body weight (nearest $0.1 \mathrm{~kg}$ ) of participants, wearing lightweight clothing, was measured along with their height (nearest $0.1 \mathrm{~cm}$ ) by using an automatic extensometer (BSM 330; Biospace, Seoul, South Korea). BMI was calculated as the ratio of weight $(\mathrm{kg})$ to height squared $\left(\mathrm{m}^{2}\right)$. To assess body composition, skeletal muscle mass, fat mass, and fat percentage were measured using a bioelectrical impedance analyzer (ACCUNIQ BC720; SELVAS Healthcare Inc., Daejeon, South Korea). Waist circumference was measured at the horizontal plane midway between the lowest ribs and the iliac crest with the participant in a standing position.

Fasting blood samples were collected from an antecubital vein to assess metabolic parameters at baseline and after the 8 -week intervention. White blood cell (WBC) counts were quantified with an XN-9000 hematology analyzer (Sysmex, IL, USA). Fasting glucose, high-sensitivity C-reactive protein, total cholesterol, triglyceride, and HDL cholesterol levels were measured with the ADVIA 1650 Clinical Chemistry system (Siemens Medical Solutions, Tarrytown, NY, USA). Unless triglycerides were $>400 \mathrm{mg} / \mathrm{dL}$, low-density lipoprotein (LDL) cholesterol levels were calculated using the Friedewald equation as follows: $\mathrm{LDL}$ cholesterol $=$ total cholesterol $-\mathrm{HDL}$ cholesterol - triglyceride $/ 5 .{ }^{23}$ Fasting insulin was measured by an electrochemiluminescence immunoassay using an Elecsys 2010 instrument (Roche, Indianapolis, IN, USA). Insulin resistance was estimated using the homeostasis model assessment of insulin resistance (HOMA-IR) method by applying the following formula: HOMA-IR $=$ fasting insulin $(\mu \mathrm{IU} / \mathrm{mL}) \times$ fasting glucose $(\mathrm{mg} / \mathrm{dL}) / 405 .^{24}$

At baseline and after the intervention, participants completed six questionnaires. The 24-h dietary recall method was used to calculate the intake of total calories and specific nutrients. The above-mentioned modified Mediterranean Diet Adherence Screener was used to assess adherence to the 
Mediterranean diet. The Godin Leisure-Time Exercise Questionnaire was used to calculate the quantity of physical activity in metabolic equivalent-hours per week. Quality of life was measured using three common questionnaires: the European Organisation for Research and Treatment of Cancer quality of life questionnaire (EORTC QLQ-C30), the obesity-related problems (OP) scale, and the Functional Assessment of Chronic Illness Therapy-Fatigue (FACIT-F) subscale. The EORTC QLQ-C30 comprises 30 items, including global health status, five functional scales, and eight symptom scales. ${ }^{25}$ High scores on global health status and five functional scales indicate a better quality of life. The OP scale primarily focuses on the impact of obesity on psychosocial functioning, and higher scores indicate more problems. ${ }^{26}$ The FACIT-F subscale uses 13 items to measure fatiguerelated difficulty in managing daily activities, with higher scores indicating less fatigue and a better quality of life. ${ }^{27}$

Safety assessments consisted of evaluations of treatment emergent adverse events, concomitant medications, vital signs (recorded at each visit), and clinical laboratory measures (recorded at baseline and at week 8), including serum creatinine and liver function tests.

\section{Statistical Analysis}

All data are presented as the medians (interquartile ranges). Differences in baseline characteristics between the intervention groups were analyzed on the intention-to-treat population using the Kruskal-Wallis test. The primary and secondary endpoints were analyzed on the per-protocol population. Within-group differences after the intervention were analyzed by the Wilcoxon's signed-rank test, and differences between groups postintervention were analyzed using the Quade's rank analysis of covariates, adjusted for age and initial BMI. A $P$ value of $<0.05$ was considered statistically significant. All analyses were performed using SPSS for Windows (version 23.0; SPSS Inc., Chicago, Ill., USA). The datasets used and/or analyzed during the present study are available from the corresponding author on reasonable request.

\section{Results}

\section{Baseline Characteristics}

A total of 75 candidates were screened for eligibility, and 72 participants (44 breast cancer survivors and 28 non-cancer patients) were enrolled in the study. The rate of study discontinuation was 22.2\% (MeDiet+NB, 29.4\%; MeDiet-only, $4.8 \%$ ). In total, 56 participants finally completed the study (Figure 1). The baseline characteristics of participants are shown in Table 1. We found no significant differences between the groups with regard to age, body weight, or metabolic parameters at baseline. However, BMIs differed at baseline

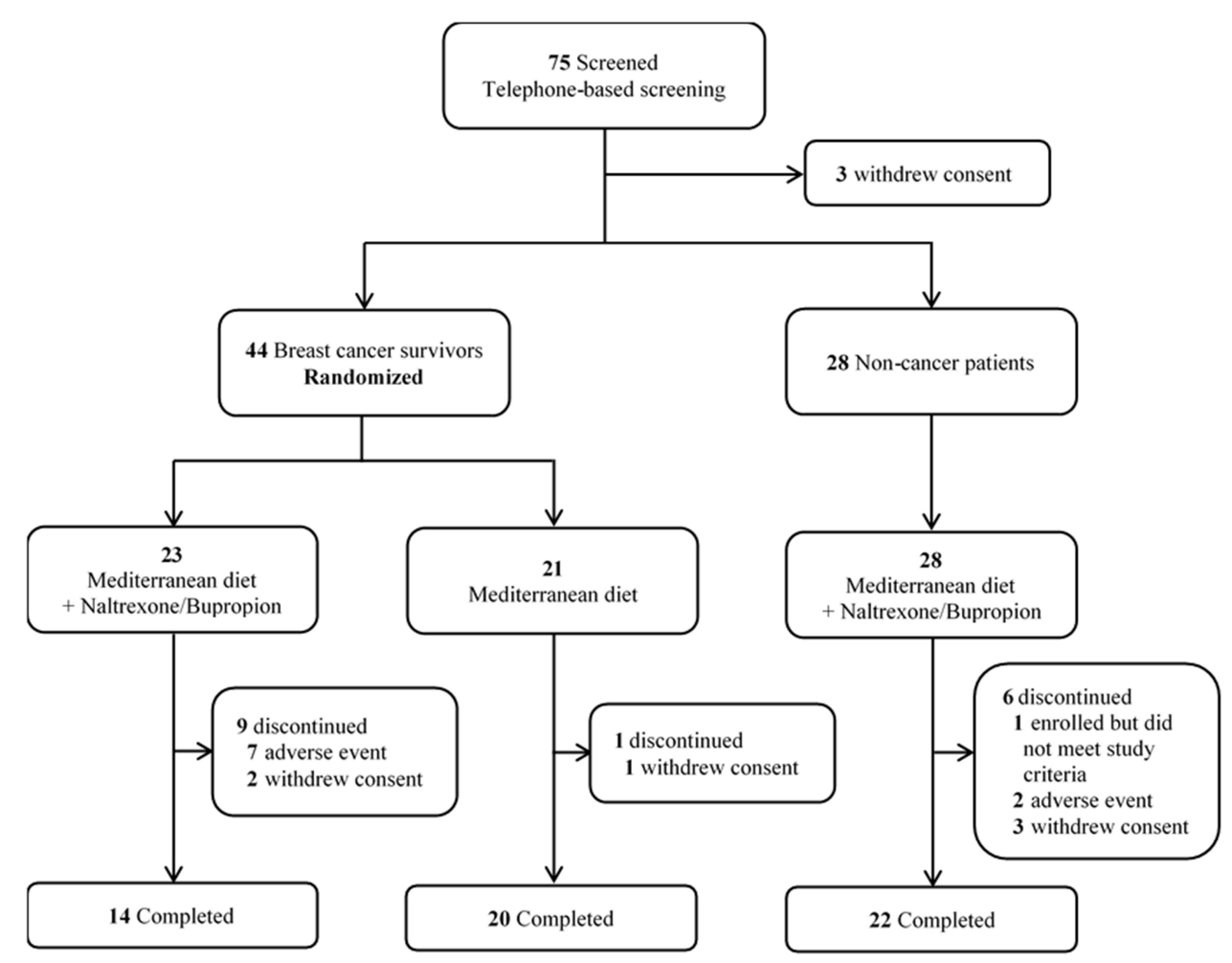

Figure I Flow chart of study participant selection. 
between the groups; therefore, we analyzed the outcomes after adjusting for initial BMI.

\section{Adherence to the Dietary Intervention}

After the 8-week intervention, the percentages of participants with high adherence to the Mediterranean diet (score of $\geq 9$ ) increased in all three groups (Figure 2). Changes in nutrient intake measured by 24-h dietary recall method are listed in Table 2 . Monounsaturated fatty acid/saturated fatty acid ratio and dietary fiber intake increased after 8 weeks $(P=0.046$ and $P<0.001$, respectively), and the median total calorie intake decreased to $<1500 \mathrm{kcal}$ per day, which reflects high adherence to the Mediterranean diet. The comparison of changes in nutrient intake among the study groups revealed no significant differences, since the same dietary intervention was advised to all three groups (Online supplementary Table

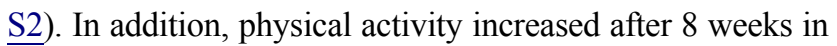
all participants $(\mathrm{P}<0.001)$ (Table 2$)$, although the changes did not differ among groups (Table S2).

\section{Endpoints}

Table 3 shows the changes in body weight, body composition, and metabolic parameters after the intervention. Body weight, BMI, and fat mass decreased in all three groups after 8 weeks $(P<0.05$ versus baseline by Wilcoxon's signed-rank test). Quade's rank analysis of covariates, adjusted for age and initial BMI, was performed to estimate the statistical differences between groups. However, there were no significant differences among the three groups or between the MeDiet $+\mathrm{NB}$ and MeDiet-only groups.

Table I Baseline Characteristics of the Study Participants

\begin{tabular}{|c|c|c|c|c|}
\hline \multirow[t]{3}{*}{ Characteristics } & \multicolumn{2}{|c|}{ Breast Cancer Survivors } & \multirow{2}{*}{$\begin{array}{l}\text { Non-Cancer Patients } \\
\text { MeDiet+NB }\end{array}$} & \multirow[t]{3}{*}{$P$ value $^{a}$} \\
\hline & MeDiet+NB & MeDiet Only & & \\
\hline & $(n=23)$ & $(n=21)$ & $(n=28)$ & \\
\hline Age (years) & $56.0(52.0,57.5)$ & $55.0(50.5,58.8)$ & $53.5(49.5,57.0)$ & 0.329 \\
\hline \multicolumn{5}{|l|}{ Body composition } \\
\hline Weight (kg) & $66.7(63.1,7 I . I)$ & $65.7(61.7,69.1)$ & $72.0(64.5,80.1)$ & 0.076 \\
\hline BMI $\left(\mathrm{kg} / \mathrm{m}^{2}\right)$ & $27.6(25.7,28.6)$ & $26.2(24.9,27.4)$ & $29.2(25.7,31.4)$ & 0.025 \\
\hline Skeletal muscle (kg) & $22.7(21.1,23.9)$ & $22.0(21.4,22.8)$ & $23.1(21.1,25.9)$ & 0.459 \\
\hline Fat mass $(\mathrm{kg})$ & $24.8(22.9,28.5)$ & $23.6(21.0,28.6)$ & $29.1(23.3,32.3)$ & 0.083 \\
\hline Fat percentage & $37 . I(36.3,4 I .2)$ & $37.1(34.7,42.4)$ & $38.7(35.2,43.5)$ & 0.329 \\
\hline WHR & $0.91(0.90,1.00)$ & $0.91(0.87,0.98)$ & $0.92(0.88,1.00)$ & 0.604 \\
\hline \multicolumn{5}{|l|}{ Diet and physical activity } \\
\hline Total calorie (kcal/day) & I744.8 (| |88.7, 2068.0) & $1653.8(\mid 321.6,1883.4)$ & | 449.8 (|295.2, | 1999.8) & 0.901 \\
\hline Mediterranean diet score & $8.0(5.5,10.0)$ & $8.0(7.0,9.8)$ & $7.0(4.5,9.0)$ & 0.259 \\
\hline GLTEQ score (MET-h/wk) & $9.0(0.0,26.5)$ & $6.0(3.0,14.5)$ & $4.8(0.0,17.5)$ & 0.642 \\
\hline \multicolumn{5}{|l|}{ Metabolic parameters } \\
\hline 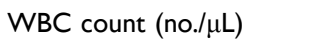 & $5.55(4.93,6.19)$ & $5.15(4.40,5.78)$ & $5.25(4.46,6.26)$ & 0.597 \\
\hline Fasting glucose (mg/dL) & $88.0(82.5,105.5)$ & $85.5(79.0,97.5)$ & $86.0(82.0,97.5)$ & 0.643 \\
\hline Insulin $(\mu \mathrm{lU} / \mathrm{mL})$ & $7.50(5.15,12.00)$ & $7.45(5.33,9.58)$ & $7.90(5.05,9.80)$ & 0.880 \\
\hline HOMA-IR & $1.52(1.08,2.78)$ & $1.49(1.19,2.25)$ & $1.70(I .06,2.5 I)$ & 0.804 \\
\hline Total cholesterol (mg/dL) & $196.0(153.5,209.5)$ & $196.5(182.3,219.0)$ & $193.0(165.5,207.5)$ & 0.478 \\
\hline Triglyceride (mg/dL) & $136.0(|2| .0,186.5)$ & $124.0(92.5,193.5)$ & II $2.0(87.0$, I 45.5$)$ & 0.140 \\
\hline HDL cholesterol (mg/dL) & $56.6(49.8,59.5)$ & $54.0(47.5,64.1)$ & $64.0(47.7,71.7)$ & 0.262 \\
\hline LDL cholesterol (mg/dL) & $109.9(63.5,130.1)$ & II $3.5(99.0,132.7)$ & II 0.1 (84.8, I24.3) & 0.537 \\
\hline \multicolumn{5}{|l|}{ Vital signs } \\
\hline Systolic BP (mmHg) & $121.0(117.5,125.5)$ & $127.5(119.8,140.8)$ & $123.0(116.5,133.5)$ & 0.173 \\
\hline Diastolic BP (mmHg) & $79.0(77.5,84.0)$ & $87.0(77.3,97.3)$ & $79.0(74.0,85.0)$ & 0.143 \\
\hline Heart rate (beats/min) & $72.0(66.5,83.5)$ & $75.0(68.3,80.8)$ & $75.0(67.0,84.0)$ & 0.812 \\
\hline
\end{tabular}

Notes: Data are expressed as medians (interquartile ranges). ${ }^{a} P$ values calculated by the Kruskal-Wallis test.

Abbreviations: BMI, body mass index; BP, blood pressure; GLTEQ, Godin Leisure-Time Exercise Questionnaire; HDL, high-density lipoprotein; HOMA-IR, homeostasis model of assessment-insulin resistance; LDL, low-density lipoprotein; MeDiet, Mediterranean diet; MET-h/wk, metabolic equivalents-hour/week; NB, naltrexone/bupropion; WBC, white blood cell; WHR, waist-hip ratio. 


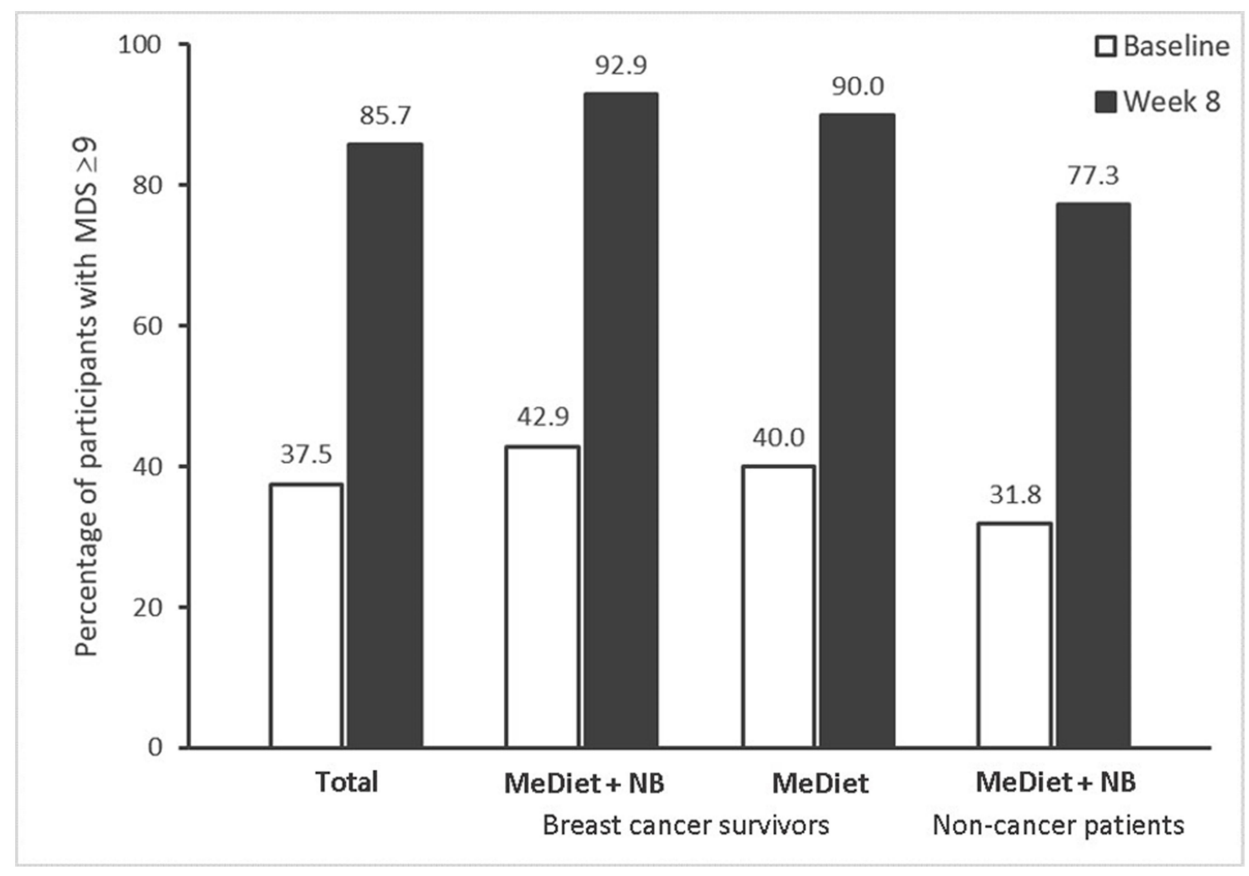

Figure 2 Percentages of participants with a Mediterranean diet score (MDS) $\geq 9$ by group.

Among measures of metabolic parameters, fasting glucose, insulin, and HOMA-IR significantly decreased in all three groups $(P<0.05$ versus baseline by Wilcoxon's signed-rank test) (Table 3). In addition, WBC count, total cholesterol, triglyceride, and LDL cholesterol were slightly reduced in all three groups, some with statistically significant

Table 2 Changes in Nutrient Intake and Physical Activity After Intervention

\begin{tabular}{|l|l|l|l|}
\hline Nutrient Intake & Baseline & Week 8 & P value $^{\mathbf{a}}$ \\
\hline Total Calorie & $1653.8(1308.1$, & $1386.2(1133.0$, & $0.00 I$ \\
(kcal) & $1967.0)$ & $1614.9)$ & \\
Carbohydrate (\%) & $54.5(42.8,62.3)$ & $54.6(47.5,60.9)$ & 0.519 \\
Protein (\%) & $15.9(14.2,17.7)$ & $15.9(13.9,20.2)$ & 0.405 \\
Total fat (\%) & $28.3(22.4,34.7)$ & $28.4(24.1,35.2)$ & 0.807 \\
SFA (\%) & $5.2(3.2,7.2)$ & $5.7(4.0,7.2)$ & 0.967 \\
MUFA (\%) & $6.5(4.8,9.1)$ & $8.3(5.6,10.5)$ & 0.267 \\
PUFA (\%) & $8.2(4.8,10.7)$ & $9.0(4.8,13.4)$ & 0.332 \\
n-3 PUFA (\%) & $0.43(0.05,0.84)$ & $0.57(0.12,1.31)$ & 0.209 \\
n-6 PUFA (\%) & $2.39(0.65,5.13)$ & $3.17(0.79,6.67)$ & 0.212 \\
MUFA/SFA ratio & $1.24(1.09,1.51)$ & $1.35(1.13,1.84)$ & 0.046 \\
PUFA/SFA ratio & $1.75(0.90,2.45)$ & $1.64(1.02,2.28)$ & 0.832 \\
Dietary fiber (g/ & $13.5(8.8,17.9)$ & $18.2(11.9,23.3)$ & $<0.001$ \\
I000 kcal) & & & \\
GLTEQ score & $7.1(2.0,18.0)$ & $15.8(4.7,30.0)$ & $<0.001$ \\
\hline
\end{tabular}

Notes: Data are expressed as medians (interquartile ranges). ${ }^{a} P$ values calculated by Wilcoxon's signed-rank test.

Abbreviations: MUFA, monounsaturated fatty acid; PUFA, polyunsaturated fatty acid; SFA, saturated fatty acid; GLTEQ, Godin Leisure-Time Exercise Questionnaire. within-group changes. Among the groups, only triglyceride levels were significantly different $(P=0.025$ by Quade's rank analysis of covariates). Other metabolic parameters were not significantly different among the three groups or between the MeDiet+NB and MeDiet-only groups.

In all participants, quality of life as assessed by selfreported questionnaires improved after the intervention (Figure 3). Global health status and five functional scales, as assessed by the EORTC QLQ-C30, significantly increased after 8 weeks $(P<0.05$ by Wilcoxon's signed-rank test). The FACIT-F subscale also increased $(P=0.002)$ and OP scale decreased $(P=0.031)$, indicating quality of life improvements. No significant differences in quality of life were found among the groups (Online supplementary Table S3).

\section{Safety and Tolerability}

Vital signs, including blood pressure and heart rate, were in the normal or prehypertension range at baseline in all three groups (Table 1). After 8 weeks, systolic blood pressure significantly decreased in the cancer survivor MeDiet-only group and the non-cancer MeDiet+NB group $(P<0.05$ by Wilcoxon's signed-rank test, data not shown). Diastolic blood pressure significantly decreased only in the non-cancer patient group, and heart rate showed no significant change in all three groups. Clinical laboratory measures, including serum creatinine and liver function tests, showed no evidence of treatment-related hepatotoxicity or nephrotoxicity (data not shown). 
Table 3 Changes in Body Weight, Body Composition, and Metabolic Parameters After Intervention

\begin{tabular}{|c|c|c|c|c|c|}
\hline \multirow[t]{3}{*}{ Parameters } & \multicolumn{2}{|c|}{ Breast Cancer Survivors } & \multirow{2}{*}{$\begin{array}{l}\text { Non-Cancer Patients } \\
\text { MeDiet+NB }\end{array}$} & \multirow[t]{3}{*}{$P$ value ${ }^{a}$} & \multirow[t]{3}{*}{$P$ value $^{b}$} \\
\hline & MeDiet+NB & MeDiet Only & & & \\
\hline & $(n=14)$ & $(n=20)$ & $(n=22)$ & & \\
\hline Weight (kg) & $-2.8(-3.6,-1.9)^{*}$ & $-1.8(-3.3,-0.6)^{*}$ & $-2.5(-4.2,-1.8)^{*}$ & 0.394 & 0.209 \\
\hline BMI $\left(\mathrm{kg} / \mathrm{m}^{2}\right)$ & $-1.1(-1.5,-0.7)^{*}$ & $-0.7(-1.4,-0.2)^{*}$ & $-1.0(-1.7,-0.7)^{*}$ & 0.404 & 0.212 \\
\hline Skeletal muscle (kg) & $-0.1(-0.8,0.3)$ & $-0.4(-0.8,0.6)$ & $-0.2(-1.2,0.1)^{*}$ & 0.938 & 0.867 \\
\hline Fat mass $(\mathrm{kg})$ & $-1.7(-3.2,-0.8)^{*}$ & $-1.8(-2.7,-0.6)^{*}$ & $-2.3(-3.0,-0.1)^{*}$ & 0.771 & 0.469 \\
\hline Fat percentage (\%) & $-1.4(-3.6,0.3)^{*}$ & $-1.4(-2.7, I .1)$ & $-1.6(-2.9,-0.4)^{*}$ & 0.623 & 0.393 \\
\hline WHR & $-0.02(-0.05,0.01)^{*}$ & $-0.01(-0.04,0.02)$ & $-0.01(-0.04,-0.01)^{*}$ & 0.386 & 0.169 \\
\hline 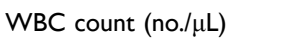 & $-0.01(-0.69,0.78)$ & $-0.66(-0.94,0.10)^{*}$ & $-0.01(-0.74,1.06)$ & 0.503 & 0.241 \\
\hline Fasting glucose (mg/dL) & $-10.0(-18.5,-6.0)^{*}$ & $-7.0(-13.0,-3.0)^{*}$ & $-12.0(-15.0,-6.5)^{*}$ & 0.627 & 0.332 \\
\hline Insulin $(\mu \mathrm{IU} / \mathrm{mL})$ & $-2.60(-4.15,0.25)^{*}$ & $-1.10(-2.10,-0.10)^{*}$ & $-1.70(-2.85,-0.05)^{*}$ & 0.467 & 0.455 \\
\hline HOMA-IR & $-0.65(-1.09,-0.13)^{*}$ & $-0.40(-0.50,-0.17)^{*}$ & $-0.41(-0.99,-0.14)^{*}$ & 0.589 & 0.595 \\
\hline Total cholesterol (mg/dL) & $-23.0(-32.5,-0.5)^{*}$ & $-5.0(-23.0,7.0)$ & $-18.0(-24.0,3.5)^{*}$ & 0.461 & 0.245 \\
\hline Triglyceride (mg/dL) & $-41.0(-59.0,-23.0)^{*}$ & $-25.0(-57.0,8.0)^{*}$ & $-4.0(-26.0,28.0)$ & 0.025 & 0.703 \\
\hline HDL cholesterol (mg/dL) & $0.4(-1.2,6.2)$ & $0.4(-2.2,7.8)$ & $-0.7(-5.0,8.6)$ & 0.735 & 0.763 \\
\hline LDL cholesterol (mg/dL) & $-16.6(-24.9,5.2)$ & $-7.7(-17.6,9.1)$ & $-10.4(-27.2,-0.2)^{*}$ & 0.274 & 0.139 \\
\hline
\end{tabular}

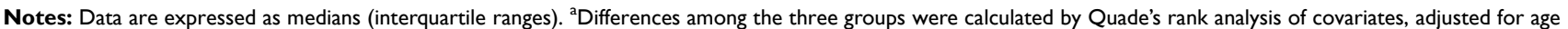
and initial BMI. 'Differences between the MeDiet+NB and MeDiet-only groups were calculated by Quade's rank analysis of covariates, adjusted for age and initial BMI. $* P$ value $<0.05$ vs baseline values by Wilcoxon's signed-rank test.

Abbreviations: BMI, body mass index; HDL, high-density lipoprotein; HOMA-IR, homeostasis model of assessment-insulin resistance; LDL, low-density lipoprotein; MeDiet, Mediterranean diet; NB, naltrexone/bupropion; WBC, white blood cell; WHR, waist-hip ratio.

Of the 51 participants who received the naltrexone and bupropion treatment, 39 participants (76\%) experienced adverse effects during the trial. Nausea was the most frequent adverse effect (39\%), followed by dizziness $(33 \%)$, dry mouth $(25 \%)$, and constipation (22\%). The symptoms were mostly mild to moderate in intensity. One serious adverse effect, syncope, occurred in the noncancer patient group, which was considered unlikely related to the study drug. Nine participants discontinued the study due to adverse effects, including nausea $(10 \%)$, urticaria $(4 \%)$, dizziness $(2 \%)$, and syncope $(2 \%)$.

\section{Discussion}

This study is the first to report the combined effect of a Mediterranean diet and naltrexone/bupropion treatment on body weight, metabolic parameters, and quality of life in breast cancer survivors who are overweight or obese, known risk factors for cancer recurrence and mortality. The 8-week interventions reduced body weights, BMI, and fat mass, while improving metabolic parameters and quality of life in all participants. However, no significant difference among the three groups was observed.

Unexpectedly, we found no additional effect of naltrexone/ bupropion medication on body weight and metabolic parameters despite evidence from previous studies showing effective weight reduction with these drugs. ${ }^{28,29}$ This discrepancy may be due to the low dose of naltrexone/bupropion used in this study. Unlike previous studies using the approved maximum doses of naltrexone (32 mg) and bupropion (360 mg), only half of the doses were used in this study. The open-label aspect of the study without a placebo could also be a reason. An increase in physical activity was seen in the MeDiet-only group, which could suggest that the participants in this group made more efforts to achieve lifestyle modifications because they did not take the obesity drugs.

Although this pilot study could not accurately evaluate the effectiveness of the Mediterranean diet since there was no control group without the Mediterranean diet, our results are consistent with previous randomized clinical trials demonstrating the benefits of the Mediterranean diet for body weight and metabolic parameters. $^{30,31}$ The PREDIMED study group reported reduced fasting glucose, insulin, and insulin resistance as short-term effects on cardiovascular risk factors of the Mediterranean diet, compared to a low-fat diet, ${ }^{32}$ which is in agreement with findings obtained in our study. Monounsaturated fats from olive oil, an important component of the Mediterranean diet, have been shown to help improve glucose metabolism and insulin sensitivity, as well as increase postprandial fat oxidation. ${ }^{33,34}$ The effects of the Mediterranean diet on body weight and composition are attributed to a low consumption of 

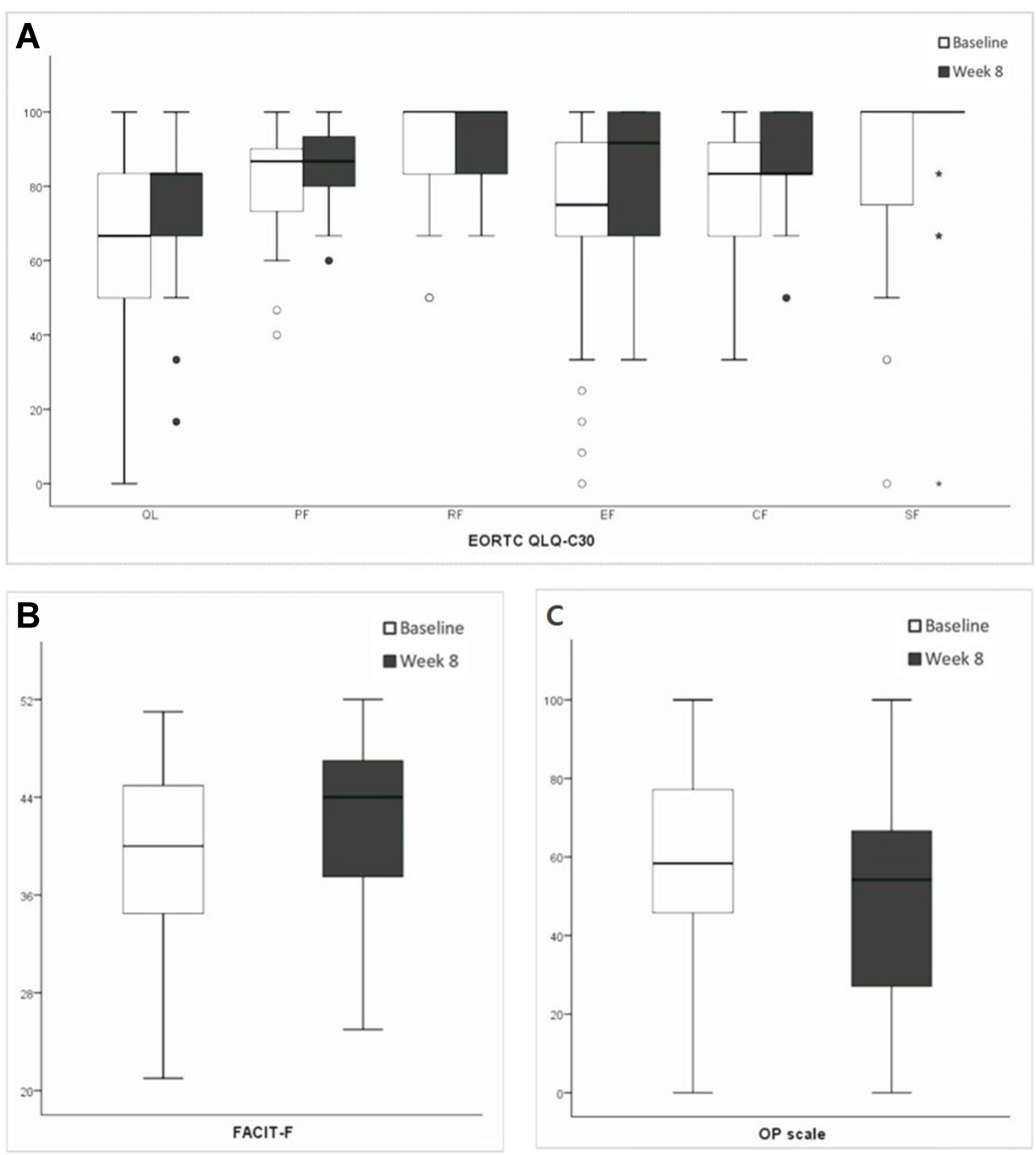

Figure 3 Changes in quality of life as assessed by questionnaires. (A) Global health status (QL) and five functional scales, namely, physical functioning (PF), role functioning (RF), emotional functioning (EF), cognitive functioning (CF), and social functioning (SF), of EORTC QLQ-C30 at baseline and week 8. (B) FACIT-F subscale at baseline and week 8. (C) OP scale at baseline and week 8.

animal products and high consumption of vegetables, fruits, and legumes. This diet is not energy dense, has a low glycemic load, and has large amounts of vitamins and dietary fiber, ${ }^{35}$ which was confirmed by our data.

In the present study, we also found that there were no differences in the results for breast cancer survivors and noncancer obese people: only the dropout rate due to the adverse events of the obesity drug was higher in breast cancer survivors. This may be because the breast cancer survivors could feel more anxious about adverse events, such as nausea, than non-cancer people. To our knowledge, this is the first weight loss intervention using obesity drugs in breast cancer survivors, while the benefits of the Mediterranean diet for breast cancer patients are relatively well known. Oleic acid in olive oil has been shown to regulate oncogenes in human breast cancer cells. ${ }^{36}$ Moreover, oleocanthal, a polyphenol in olive oil, has been found to inhibit the proliferation, invasion, and growth of tumor cells in vitro and in breast cancer models in vivo. ${ }^{37}$ Therefore, the Mediterranean diet appears to be helpful as a dietary intervention in breast cancer patients. Further studies about the choice of obesity drugs to breast cancer patients are warranted.

The present study has several limitations. First, the use of an 8-week intervention precluded investigations of the effectiveness of the intervention on weight maintenance or cancer recurrence. Second, we recruited non-cancer patients matched to survivors by age, not BMI. As a result, the baseline BMIs differed between groups, requiring adjustments for this factor in statistical analyses. Third, we relied on self-reported dietary intake using the 
24-h dietary recall method, which may have recall bias. However, the increased monounsaturated fatty acid/saturated fatty acid ratio and dietary fiber measurements by 24-h dietary recall method were consistent with consumption of a Mediterranean diet. Finally, the rate of study discontinuation was higher than the anticipated dropout rate of $10 \%$. Most of the participants reported dropping out due to the adverse effects of the naltrexone/bupropion medication.

\section{Conclusions}

In summary, we found that the Mediterranean diet, with or without naltrexone/bupropion treatment, facilitates weight loss, improves metabolic parameters, and increases quality of life in breast cancer survivors who are overweight or obese. The combination of the Mediterranean diet with naltrexone/bupropion treatment did not produce superior changes in outcomes when compared to the Mediterranean diet alone. Future studies should include a larger number of participants over a longer period of time to determine the effect of the Mediterranean diet on breast cancer recurrence or survival.

\section{Abbreviations}

BMI, body mass index; EORTC QLQ-C30, European Organisation for Research and Treatment of Cancer Quality of Life Questionnaire; FACIT-F, Functional Assessment of Chronic Illness Therapy-Fatigue; HDL, high-density lipoprotein; HOMA-IR, homeostasis model assessment of insulin resistance; LDL, low-density lipoprotein; MeDiet, Mediterranean diet; NB, naltrexone/ bupropion; OP, obesity-related problems; PREDIMED, Prevención con Dieta Mediterránea; WBC, white blood cell.

\section{Data Sharing Statement}

The datasets used and/or analyzed during the current study are available from the corresponding author on reasonable request.

\section{Ethics Approval and Consent to Participate}

The study was approved by the Institutional Review Board of Gangnam Severance Hospital (IRB number 3-20170097). Written informed consent was obtained from each participant before screening and data collection. All datasets intended for sharing are deidentified. All procedures were conducted in accordance with the ethical standards of the 1975 Helsinki Declaration.

\section{Acknowledgments}

We thank all the researchers and subjects who participated in this study.

\section{Author Contributions}

All authors made a significant contribution to the work reported, whether that is in the conception, study design, execution, acquisition of data, analysis and interpretation, or in all these areas; took part in drafting, substantially revising, or critically reviewing the article; gave final approval of the version to be published; have agreed on the journal to which the article will be submitted; and have agreed to take responsibility and be accountable for all aspects of the article.

\section{Funding}

This research was supported by the Bio \& Medical Technology Development Program, through the National Research Foundation of Korea funded by the Ministry of Science and ICT (NRF-2013M3A9B6046416, NRF2018R1D1A1B07049223). This work was supported by the Technology Innovation Program (20002781, A Platform for Prediction and Management of Health Risk Based on Personal Big Data and Lifelogging) funded by the Ministry of Trade, Industry \& Energy (MOTIE, Korea).

\section{Disclosure}

We declare no competing interests for this work.

\section{References}

1. Jung KW, Won YJ, Kong HJ, Lee ES. Cancer statistics in Korea: incidence, mortality, survival, and prevalence in 2015. Cancer Res Treat. 2018;50(2):303-316. doi:10.4143/crt.2018.143

2. Biganzoli E, Desmedt C, Fornili M, et al. Recurrence dynamics of breast cancer according to baseline body mass index. Eur J Cancer. 2017;87:10-20. doi:10.1016/j.ejca.2017.10.007

3. Picon-Ruiz M, Morata-Tarifa C, Valle-Goffin JJ, Friedman ER, Slingerland JM. Obesity and adverse breast cancer risk and outcome: mechanistic insights and strategies for intervention. CA Cancer J Clin. 2017;67(5):378-397.

4. Kroes M, Osei-Assibey G, Baker-Searle R, Huang J. Impact of weight change on quality of life in adults with overweight/obesity in the United States: a systematic review. Curr Med Res Opin. 2016;32 (3):485-508. doi:10.1185/03007995.2015.1128403

5. Fuster JJ, Ouchi N, Gokce N, Walsh K. Obesity-induced changes in adipose tissue microenvironment and their impact on cardiovascular disease. Circ Res. 2016;118(11):1786-1807. doi:10.1161/CIRCRESA HA.115.306885 
6. Runowicz CD, Leach CR, Henry NL, et al. American Cancer Society/ American Society of Clinical Oncology Breast cancer survivorship care guideline. $J$ Clin Oncol. 2016;34(6):611-635. doi:10.1200/ JCO.2015.64.3809

7. Rock CL, Flatt SW, Byers TE, et al. Results of the Exercise and Nutrition to Enhance Recovery and Good Health for You (ENERGY) Trial: a behavioral weight loss intervention in overweight or obese breast cancer survivors. J Clin Oncol. 2015;33(28):3169-3176. doi:10.1200/JCO.2015.61.1095

8. Harrigan M, Cartmel B, Loftfield E, et al. Randomized trial comparing telephone versus in-person weight loss counseling on body composition and circulating biomarkers in women treated for breast cancer: the Lifestyle, Exercise, and Nutrition (LEAN) study. J Clin Oncol. 2016;34(7):669-676. doi:10.1200/JCO.2015.61.6375

9. Jensen MD, Ryan DH, Apovian CM, et al. 2013 AHA/ACC/TOS guideline for the management of overweight and obesity in adults: a report of the American College of Cardiology/American Heart Association task force on practice guidelines and the obesity society. $J$ Am Coll Cardiol. 2014;63(25Pt B):2985-3023. doi:10.1016/j.jacc.2013.11.004

10. Estruch R, Ros E, Salas-Salvadó J, et al. Primary prevention of cardiovascular disease with a mediterranean diet. $N$ Engl J Med. 2013;368(14):1279-1290. doi:10.1056/NEJMoa1200303

11. Becerra-Tomás N, Blanco Mejía S, Viguiliouk E, et al. Mediterranean diet, cardiovascular disease and mortality in diabetes: a systematic review and meta-analysis of prospective cohort studies and randomized clinical trials. Crit Rev Food Sci Nutr. 2020;60(7):1207-1227. doi:10.1080/10408398.2019.1565281

12. Schwingshackl L, Schwedhelm C, Galbete C, Hoffmann G. Adherence to mediterranean diet and risk of cancer: an updated systematic review and meta-analysis. Nutrients. 2017;9(10):10. doi:10.3390/nu9101063

13. Toledo E, Salas-Salvadó J, Donat-Vargas C, et al. Mediterranean diet and invasive breast cancer risk among women at high cardiovascular risk in the PREDIMED trial: a randomized clinical trial. JAMA Intern Med. 2015;175(11):1752-1760. doi:10.1001/jamainternmed.2015.4838

14. Greenway FL, Fujioka K, Plodkowski RA, et al. Effect of naltrexone plus bupropion on weight loss in overweight and obese adults (COR-I): a multicentre, randomised, double-blind, placebo-controlled, Phase 3 trial. Lancet. 2010;376(9741):595-605. doi:10.1016/S0140-6736(10)60888-4

15. Zagon IS, Donahue R, McLaughlin PJ. Targeting the opioid growth factor: opioid growth factor receptor axis for treatment of human ovarian cancer. Exp Biol Med (Maywood). 2013;238(5):579-587. doi: $10.1177 / 1535370213488483$

16. Li Z, You Y, Griffin N, Feng J, Shan F. Low-dose naltrexone (LDN): a promising treatment in immune-related diseases and cancer therapy. Int Immunopharmacol. 2018;61:178-184. doi:10.1016/j.intimp.2018.05.020

17. Nuñez GR, Pinczowski H, Zanellato R, et al. Bupropion for control of hot flashes in breast cancer survivors: a prospective, double-blind, randomized, crossover, pilot Phase II trial. J Pain Symptom Manage. 2013;45(6):969-979. doi:10.1016/j.jpainsymman.2012.06.011

18. Mathias C, Cardeal Mendes CM, Pondé de Sena E, et al. An open-label, fixed-dose study of bupropion effect on sexual function scores in women treated for breast cancer. Ann Oncol. 2006;17(12):1792-1796. doi:10.1093/annonc/mdl304

19. WHO expert consultation. Appropriate body-mass index for Asian populations and its implications for policy and intervention strategies. Lancet. 2004;363(9403):157-163. doi:10.1016/S0140-6736(03)15268-3

20. Schröder H, Fitó M, Estruch R, et al. A short screener is valid for assessing mediterranean diet adherence among older Spanish men and women. J Nutr. 2011;141(6):1140-1145. doi:10.3945/jn.110.135566

21. Simapivapan P, Boltong A, Hodge A. To what extent is alcohol consumption associated with breast cancer recurrence and second primary breast cancer?: A systematic review. Cancer Treat Rev. 2016;50:155-167. doi:10.1016/j.ctrv.2016.09.010
22. Cho JW, Kweon MR, Park YM, et al. A survey of diabetic educators and patients for the revision of korean food exchange lists. Diabetes Metab J. 2011;35(2):173-181. doi:10.4093/dmj.2011.35.2.173

23. Friedewald WT, Levy RI, Fredrickson DS. Estimation of the concentration of low-density lipoprotein cholesterol in plasma, without use of the preparative ultracentrifuge. Clin Chem. 1972;18(6):499-502. doi:10.1093/clinchem/18.6.499

24. Matthews DR, Hosker JP, Rudenski AS, Naylor BA, Treacher DF, Turner RC. Homeostasis model assessment: insulin resistance and beta-cell function from fasting plasma glucose and insulin concentrations in man. Diabetologia. 1985;28(7):412-419. doi:10.1007/BF00280883

25. Yun YH, Park YS, Lee ES, et al. Validation of the Korean version of the EORTC QLQ-C30. Qual Life Res. 2004;13(4):863-868. doi:10.1023/B:QURE.0000021692.81214.70

26. Lee YJ, Moon KH, Choi JH, Cho MJ, Shin SH, Heo Y. Validation of the Korean translation of obesity-related problems scale assessing the quality of life in obese Korean. J Korean Surg Soc. 2013;84 (3): $140-153$. doi: $10.4174 / \mathrm{jkss} .2013 .84 .3 .140$

27. Webster K, Cella D, Yost K. The Functional Assessment of Chronic Illness Therapy (FACIT) measurement system: properties, applications, and interpretation. Health Qual Life Outcomes. 2003;1(1):79. doi:10.1186/1477-7525-1-79

28. Apovian CM, Aronne L, Rubino D, et al. A randomized, phase 3 trial of naltrexone SR/bupropion SR on weight and obesity-related risk factors (COR-II). Obesity (Silver Spring). 2013;21(5):935-943. doi:10.1002/oby.20309

29. Wadden TA, Foreyt JP, Foster GD, et al. Weight loss with naltrexone $\mathrm{SR} /$ bupropion SR combination therapy as an adjunct to behavior modification: the COR-BMOD trial. Obesity (Silver Spring). 2011;19(1):110-120. doi:10.1038/oby.2010.147

30. Shai I, Schwarzfuchs D, Henkin Y, et al. Weight loss with a low-carbohydrate, mediterranean, or low-fat diet. $N$ Engl $J$ Med. 2008;359(3):229-241. doi:10.1056/NEJMoa0708681

31. Bajerska J, Chmurzynska A, Muzsik A, et al. Weight loss and metabolic health effects from energy-restricted mediterranean and Central-European diets in postmenopausal women: a randomized controlled trial. Sci Rep. 2018;8(1):11170. doi:10.1038/s41598-018-29495-3

32. Estruch R, Martínez-González MA, Corella D, et al. Effects of a mediterranean-style diet on cardiovascular risk factors: a randomized trial. Ann Intern Med. 2006;145(1):1-11. doi:10.7326/0003-4819145-1-200607040-00004

33. Due A, Larsen TM, Hermansen K, et al. Comparison of the effects on insulin resistance and glucose tolerance of 6-mo high-monounsaturated-fat, low-fat, and control diets. Am J Clin Nutr. 2008;87(4):855-862. doi:10.1093/ajen/87.4.855

34. Gillingham LG, Harris-Janz S, Jones PJ. Dietary monounsaturated fatty acids are protective against metabolic syndrome and cardiovascular disease risk factors. Lipids. 2011;46(3):209-228. doi:10.1007/s11745-0103524-y

35. Kwan HY, Chao X, Su T, et al. The anticancer and antiobesity effects of mediterranean diet. Crit Rev Food Sci Nutr. 2017;57(1):82-94. doi:10.1080/10408398.2013.852510

36. Menendez JA, Papadimitropoulou A, Vellon L, Lupu R. A genomic explanation connecting "mediterranean diet", olive oil and cancer: oleic acid, the main monounsaturated fatty acid of olive oil, induces formation of inhibitory "PEA3 transcription factor-PEA3 DNA binding site" complexes at the Her-2/neu (erbB-2) oncogene promoter in breast, ovarian and stomach cancer cells. Eur J Cancer. 2006;42 (15):2425-2432. doi:10.1016/j.ejca.2005.10.016

37. Akl MR, Ayoub NM, Mohyeldin MM, et al. Olive phenolics as c-Met inhibitors: (-)-oleocanthal attenuates cell proliferation, invasiveness, and tumor growth in breast cancer models. PLoS One. 2014;9(5): e97622. doi:10.1371/journal.pone.0097622 


\section{Publish your work in this journal}

Diabetes, Metabolic Syndrome and Obesity: Targets and Therapy is an international, peer-reviewed open-access journal committed to the rapid publication of the latest laboratory and clinical findings in the fields of diabetes, metabolic syndrome and obesity research. Original research, review, case reports, hypothesis formation, expert opinion and commentaries are all considered for publication. The manuscript management system is completely online and includes a very quick and fair peer-review system, which is all easy to use. Visit $\mathrm{http}: / /$ www.dovepress.com/testimonials.php to read real quotes from published authors. 